\title{
By-example Synthesis of Architectural Textures
}

\author{
Sylvain Lefebvre ${ }^{1,2}$ \\ Samuel Hornus ${ }^{2,3}$ \\ Anass Lasram ${ }^{2}$ \\ ${ }^{1}$ REVES / INRIA Sophia Antipolis \\ ${ }^{2}$ ALICE / INRIA Nancy \\ ${ }^{3}$ GEOMETRICA / INRIA Sophia Antipolis
}

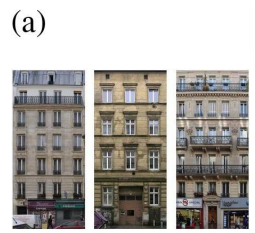

Source images
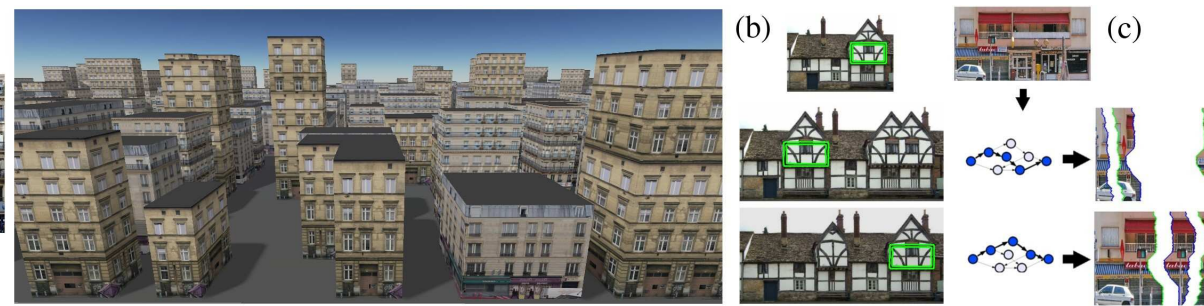

Figure 1: (a) From a source image our synthesizer generates new textures fitting surfaces of any size. (b) The user has precise control over the results. (c) Our algorithm synthesizes textures by reordering strips of the source image. Each result is a path in a graph describing the space of synthesizable images. Only the path need to be stored in memory. Decoding is performed during display, in the pixel shader.

\begin{abstract}
Textures are often reused on different surfaces in large virtual environments. This leads to unpleasing stretch and cropping of features when textures contain architectural elements. Existing retargeting methods could adapt each texture to the size of their support surface, but this would imply storing a different image for each and every surface, saturating memory. Our new texture synthesis approach casts synthesis as a shortest path problem in a graph describing the space of images that can be synthesized. Each path in the graph describes how to form a new image by cutting strips of the source image and reassembling them in a different order. Only the paths describing the result need to be stored in memory: synthesized textures are reconstructed at rendering time. The user can control repetition of features, and may specify positional constraints. We demonstrate our approach on a variety of textures, from facades for large city rendering to structured textures commonly used in video games.
\end{abstract}

\section{Introduction}

Textures are an essential primitive of Computer Graphics. They are responsible for most of the detail we perceive in computer generated images. In this paper, we focus on textures applied to urban scenery and buildings. These textures often contain a mix of materials-concrete, bricks, stones-and structure-windows, doors, lanes, control panels. We refer to such textures as architectural textures.

A typical difficulty is that architectural textures are created with a given surface size in mind. For instance, facade textures captured from photographs will only apply to buildings of the appropriate size. Door images will only apply to the corresponding door panels. Enlarging or changing the size of the surfaces results in a stretched, unpleasing and unrealistic appearance. In addition, it is often not possible to customize every texture to every surface. A first issue is of course limited authoring resources. A second, major issue comes from the extremely large quantity of data that would result from having a unique texture per surface. In the context of interactive applications the cost of such a large database of textures both in terms of storage and rendering complexity is prohibitive.

Our approach addresses both issues at once: It synthesizes, from an example, new textures fitting exactly the support surfaces and stores the result in a very compact form, typically just a few kilobytes. Results are directly used under their compact form during rendering. Decoding is simple enough to fit in a pixel shader. Using our technique, modelers only have to prepare a limited database of high quality textures: a new, unique texture resembling the original is synthesized for each surface, typically in less than a second. If the user is not satisfied with the result, several interactive controls are possible, such as explicit feature positioning through drag-anddrop. This all comes at a small cost since results are compactly stored during display, and efficiently rendered.

To achieve this, we exploit specificities of architectural textures. Our assumption is that the textures tend to be auto-similar when translated along the main axes. This is easily verified on architectural textures. However our approach only applies to this subclass of images, and will be less efficient on arbitrary images. The technique is fully automatic, unless the user wants to achieve a particular goal, such as putting a door or window at a given location. We use the self-similarities in the textures to define a generative model of the image content. In that sense, each image is turned into an image synthesizer able to generate visually similar images of any size. Once constructed, the synthesizer quickly generates textures of any width and height and outputs the result in a compact form.

The synthesizer proceeds successively in vertical and horizontal directions, the second step using the intermediate result as the source image. In each direction, it synthesizes a new image by cutting horizontal (resp. vertical) strips of the source image and pasting them side by side in a different order. This is illustrated along the $x$ direction Figure 1 (c). We search the source image for repeated content in the form of horizontal (resp. vertical) parallel paths along which similar colors are observed. Two such parallel paths are indicated by the green/blue triangles in the inset. We call these paths cuts as they define the boundaries of the strips that we "cut out" from the source image. We extract many such parallel cuts from each source image. Any pair of non-crossing cuts defines a strip, and the strips can be assembled with a number of other strips like in a puzzle (Figure 1(c)). The parallel cuts create many choices in the way strips may be assembled. 
This is the source of variety in the synthesized textures. Intuitively, given a target size our algorithm automatically determines the best choice of strips to both reach the desired size and minimize color differences along their boundaries. Despite the apparent combinatorial complexity of the problem, given a set of cuts we are able to solve efficiently for it using a graph-based formulation. The optimization reduces to a simple shortest-path computation. The solution path describes the sequence of cuts that form the boundaries of each strip; it is thus very compact.

\section{Our contributions are:}

- The formulation of the unidirectional architectural texture synthesis problem as a graph path-finding problem, together with a construction algorithm for finding cuts and building the graph for a given image (Section 3,

- An algorithm to quickly generate an image of any desired width and height, using two successive unidirectional synthesis steps (Section 4), possibly enforcing some constraints for higher visual quality or user-control over the result (Section 5 .

- A compact encoding of synthesized textures in GPU memory, together with a simple algorithm to sample them from the pixel shader (Section 6).

Applications range from large virtual city rendering to adapting structured textures to their support surfaces in video game environments (Section 7).

\section{Previous work}

Generating rich and detailed textures for virtual environments is a long standing goal of computer graphics. We present here the works most related to synthesis of architectural (structured) textures.

Procedural architectural textures A first approach to adapt textures to surfaces and reduce authoring time is to procedurally generate the textures. Early approaches for structured textures are based on cellular noise [Worley 1996]. Lefebvre and Poulin [2000] propose to learn the parameters from existing images for specific materials. Müller et al. 2007] fit a grammar on photographs of facades to deduce a generative model of their geometry. We compare this approach to ours for the specific case of facades in Section 7 Legakis et al. [2001] generate complex structured layouts of bricks and stones on the walls of buildings.

Content synthesis from example Example-based texture synthesis methods [Wei et al. 2009] generate new textures of any size resembling a small input image. Most of these methods focus on textures of unstructured, homogeneous aspect and require to precompute and store the resulting images. The approach of Sun et al. [2005] fills holes in structured images by pasting overlapping patches at discrete locations along user defined curves, minimizing for color differences. Schödl et al. [2000] synthesize infinite video loops by cutting and re-ordering pieces of an example sequence. Loops are formed by jumping backward in time to matching frames. This could be applied to images, jumping from one column to another. Compared to this scheme our graph formulation allows for arbitrarily shaped cuts and is not limited to backward jumps.

Image retargeting and reshuffling Recently, a new set of techniques have been developed to retarget images for displays of various sizes. While not exactly our goal, this could be used to adapt textures. Several approaches deform a mesh overlaid on the image, focusing stretch in areas where it is less noticeable [Gal et al.
2006; Wang et al. 2008, Cabral et al. 2009]. Instead, the seam carving approach [Avidan and Shamir 2007] iteratively removes rows and columns having low gradient in the image, one at a time. Rubinstein et al. 2009| extend the approach to compromise between stretch, crop, and seam carving.

These early methods strive to preserve all the content initially in the image. Instead, the approach of Simakov et al. [2008] summarizes the image content, discarding repetitive information. An interesting new application suggested in this work is the reshuffling of the image content, giving the ability to change the layout of features in the image. Recent approaches formulate reshuffling as a labeling problem, either at the patch level [Cho et al. 2008] or at the pixel level [Pritch et al. 2009]. These approaches are generally slow since they are iterative and based on patch matching; however they can be brought to interactivity using stochastic patch search |Barnes et al. 2009]. In all cases, the output is a new image that must be stored, hindering their use for textures in large environments.

Our goal is to provide similar functionalities for architectural textures, while affording compact storage of the result. Our approach draws inspiration from patch-based texture synthesis approaches [Kwatra et al. 2003]-we reorder pieces of the source image - and seam carving |Avidan and Shamir 2007]—we work successively with horizontal or vertical strips of the initial image. However, by relying on a graph-formulation for the choice of strips we quickly obtain results of very high quality - the best possible for the given set of cuts - and easily account for global constraints, enabling user controls. In addition, only the paths need to be recorded in memory, resulting in a very compact encoding of the results.

\section{Unidirectional synthesis}

\subsection{Overview}

Given a source image, our approach first prepares a synthesizer in a preprocessing step. The synthesizer is then used to produce visually similar images of any size. The output is a compact data structure directly used by the renderer to texture 3D models.

The image synthesizer operates in a single direction at a time, horizontally or vertically. Hence, two successive synthesis steps are necessary in order to generate images of given width and height. In the following we discuss unidirectional synthesis only, and later focus on bidirectional synthesis in Section 4 For simplicity, we describe synthesis in the horizontal direction.

In order to build a horizontal synthesizer, we first search the image for parallel vertical cuts (Section 3.4, and then build a directed graph (digraph) representing the space of all images that can be synthesized (Section 3.2). We describe first the digraph construction and synthesis step since they constitute the heart of our approach.

We introduce below the definitions used throughout the paper.

Pixels We note $[N]$ the integer interval $[0 . . N-1]$. The source image has width $W$ and height $H$. The lower left pixel has coordinates $(0,0)$.

Cuts A vertical cut $c$ is a function $[H] \mapsto\{-1\} \cup[W]$ such that $|c(y)-c(y+1)| \leq 1$ for all $y \in[H]$. We understand $c$ as a cut between the pixels in the image: for all $y$ in $[H]$ we cut the image vertically along the right boundary of pixel $(c(y), y)$ so as to separate the image in two pieces. For any cut $c$, we define $c_{\min }=$ $\min _{y \in[H]} c(y), \quad c_{\max }=\max _{y \in[H]} c(y)$ and the width $\omega\left(c, c^{\prime}\right)$ between cuts $c$ and $c^{\prime}$ as $\omega\left(c, c^{\prime}\right)=c_{\min }^{\prime}-c_{\min }$. Similar definitions apply to horizontal cuts which we do not consider further. 
Partial order on the cuts Let $\mathcal{C}$ denote a set of cuts. We define a partial order on $\mathcal{C}$ as follows. Given two cuts $c$ and $c^{\prime}$, we say that c precedes $c^{\prime}$ if and only if $c \neq c^{\prime}$ and for all $y \in[H]$ we have $c(y) \leq c^{\prime}(y)$. We write precedence as $c \prec c^{\prime}$. Two distinct cuts $c$ and $c^{\prime}$ cross each other if none precedes the other.

Given a cut $c$ (shown yellow inset) we define the successors of $c$ as $\operatorname{Succ}(\mathcal{C}, c)=\left\{c^{\prime} \in \mathcal{C} \quad \mid c \prec c^{\prime}\right\}$ (shades of green and blue inset). We say that $c \in \mathcal{C}$ is a front cut of $\mathcal{C}$ if it has no predecessor in $\mathcal{C}$. We write Front $(\mathcal{C})$ the set of front cuts of $\mathcal{C}$ (we show inset $\operatorname{Front}(\operatorname{Succ}(\mathcal{C}, c)$ ) in shades of green). Note that the cuts in $\operatorname{Front}(\mathcal{C})$ are pairwise crossing.

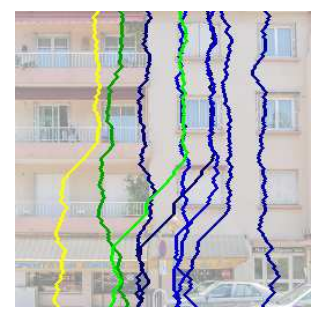

Parallels Two cuts $c$ and $c^{\prime}$ are parallel iff $c^{\prime}(y)-c(y)=\omega\left(c, c^{\prime}\right)$ for all $y \in[H]$. A cut is parallel to itself. Let $\mathcal{P}(c)$ denote the set of cuts in $\mathcal{C}$ that are parallel to $c$. Two parallel cuts are illustrated in the inset picture on the first page bottom.

Costs Each pair of parallel cuts $\left(c, c_{\|}\right)$is associated with a cost $\delta\left(c, c_{\|}\right)$. In our implementation, this cost is the sum of the color differences along the cuts, raised to a power $p$ (we use $p=4$ ):

$$
\delta\left(c, c_{\|}\right)=\sum_{y \in[H]}\left\|I(c(y)+1, y)-I\left(c_{\|}(y)+1, y\right)\right\|^{p}
$$

where $I(x, y)$ is the pixel value at $x, y$ in the image. Increasing $p$ penalizes cuts that match well overall but have a few large mismatches. Note that $\delta(c, c)=0$.

\subsection{Assembling strips}

We now describe our formulation for unidirectional synthesis. We define an assembly of strips from the source image as a sequence of cuts $A=c^{\star}, c^{0}, c_{\|}^{0}, \ldots, c^{n}, c_{\|}^{n}, c^{\dagger}$ where $c^{\star}, c^{\dagger}$ are starting and ending cuts chosen by the user (typically the first and last columns of the source image), and $c^{i}, c_{\|}^{i}$ are the ending and starting cuts of two successive strips (see Figure 2). Since they must have the same shape these two cuts are parallels: $c_{\|}^{i} \in \mathcal{P}\left(c^{i}\right) \backslash\left\{c^{i}\right\}$. The assembly quality depends on how well colors along the cuts are matching. We define the assembly cost as: $\delta(A)=\sum_{i=0}^{n} \delta\left(c^{i}, c_{\|}^{i}\right)$.

Let $\omega(A)$ be the width of the image resulting from the assembly $A$. We are searching for an assembly $A_{\text {best }}$ minimizing $\delta$ while producing an image of desired target width $\mathcal{T}$, i.e.:

$$
A_{\text {best }}=\underset{A \text { st. } \omega(A)=\mathcal{T}}{\operatorname{argmin}}(\delta(A)) .
$$

We optimize for the best possible assembly by turning the problem into a simple shortest path search in a directed graph. (We only consider directed graphs, so we will use the shorter term "graph".)

Generative graph Our reasoning for the graph formulation is as follows: Consider we add cuts - rather than strips-to the image under construction, one at a time from left to right. At any given time the partially grown image ends with a cut $c$. From this cut we are given two choices on how to continue (see Figure 2):

1. Grow the rightmost strip by adding to the image one of the cut $c^{\prime}$ located after $c$ in the source, that is a cut in $\operatorname{Succ}(\mathcal{C}, c)$. In this case, we limit the choice for $c^{\prime}$ to cuts in $\mathfrak{F}(c)=\operatorname{Front}(\operatorname{Succ}(\mathcal{C}, c))$ without loss of generality since a cut $c^{\prime} \in \operatorname{Succ}(\mathcal{C}, c)$ is either in $\mathfrak{F}(c)$ or is separated from $c$ by at least one cut in $\mathfrak{F}(c)$. This restriction on the choices for $c^{\prime}$ reduces the number of edges in the graph to be described below, roughly, from $O\left(|\mathcal{C}|^{2}\right)$ to $O(|\mathcal{C}|)$.

2. Start a new strip by jumping to a cut parallel to $c$, that is a cut in $\mathcal{P}(c) \backslash\{c\}$. This strip may be later grown.

In the first case we enlarge the image by $\omega\left(c, c^{\prime}\right)$ and add no error to the assembly. In the second case we do not enlarge the image but add an error $\delta\left(c, c_{\|}\right)$to the assembly cost.
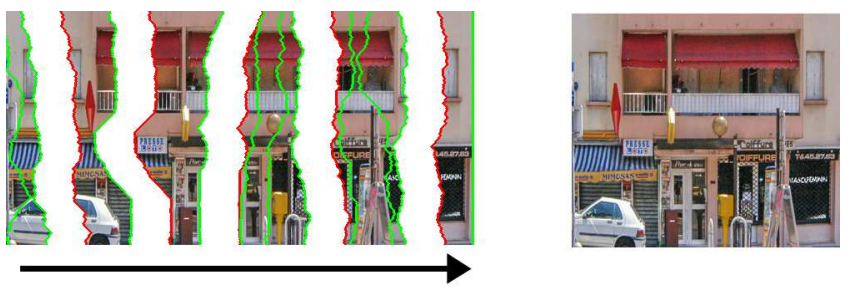

Figure 2: Left: Cuts are added one by one to the image from left to right, either growing the current strip by adding a cut (green), or starting a new strip by jumping from a cut to its parallel counterpart (red). Right: The end result, formed of six strips from the source.

We model these choices as a graph $G=\left(\mathcal{C} \times \mathbb{Z}, E_{\prec} \sqcup E_{\|}\right)$where $\sqcup$ is the disjoint union. Each node $(c, z) \in \mathcal{C} \times \mathbb{Z}$ represents the cut $c$ translated to abscissa $z$ in the result image. More precisely, the translated cut is located in the result image at $\left(c(y)-c_{\min }+z, y\right)$ for all $y \in[H]$. Thus, while the cuts are defined in the source image, the nodes of the graph are defined in the result image. The growth edges $E_{\prec}$ and the start edges $E_{\|}$capture the choices discussed above and are defined as:

$$
\begin{aligned}
E_{\prec}= & \left\{(c, z) \rightarrow\left(c^{\prime}, z+\omega\left(c, c^{\prime}\right)\right)\right. \\
& \left.c^{\prime} \in \operatorname{Front}(\operatorname{Succ}(\mathcal{C}, c)), z \in \mathbb{Z}\right\} \\
E_{\|}= & \left\{(c, z) \rightarrow\left(c^{\prime}, z\right) \quad \mid \quad c^{\prime} \in \mathcal{P}(c) \backslash\{c\}, z \in \mathbb{Z}\right\}
\end{aligned}
$$

Intuitively, edges in $E_{\prec}$ tell us that if we depart from a cut $c$ located at $z$ in the result image and grow the strip until a cut $c^{\prime} \in$ Front $(\operatorname{Succ}(S, c))$, then the next cut $c^{\prime}$ is located at $z+\omega\left(c, c^{\prime}\right)$. Edges in $E_{\|}$simply replace the current cut by its parallel counterpart, without changing the current location.

The cost of each edge $\left((c, z),\left(c^{\prime}, z^{\prime}\right)\right) \in E_{\|}$is set to $\delta\left(c, c^{\prime}\right)$ and edges in $E_{\prec}$ are given a null cost.

Paths as images We specify the start and end of the result image with two cuts $c^{\star}$ and $c^{\dagger}$. These two cuts are typically located at the left and right border of the source image but could be any other pair of cuts, possibly chosen by the user. Refer to Section 5 for more details on user controls.

Finding the best assembly for an image of size $\mathcal{T}$ thus simply amounts to finding a path in $G$ from node $\left(c^{\star}, c_{\min }^{\star}-c_{\max }^{\star}\right)$ to node $\left(c^{\dagger}, \mathcal{T}\right)$ since the assembly of strips corresponding to such a path does contain a rectangle of width $\mathcal{T}$. We use Dijkstra's algorithm to globally minimize the cost of the resulting assembly. The graph $G$ is infinite, but for a desired width $\mathcal{T}$ we need only consider a finite subgraph $G^{\prime}=\left(\mathcal{C} \times\left[c_{\text {min }}^{\star}-c_{\text {max }}^{\star} . \mathcal{T}\right], E_{\prec} \sqcup E_{\|}\right)$, because the $z$ component of the nodes along any path in $G$ form a non-decreasing sequence.

Graph traversal For space efficiency during Dijkstra's algorithm execution, we generate the graph edges implicitly from its nodes: growth edges are generated from the sets $\operatorname{Front} \operatorname{Succ}(\mathcal{C}, c))$ that we keep in memory for each cut $c \in \mathcal{C}$ (see Eq. 1). Similarly, start 


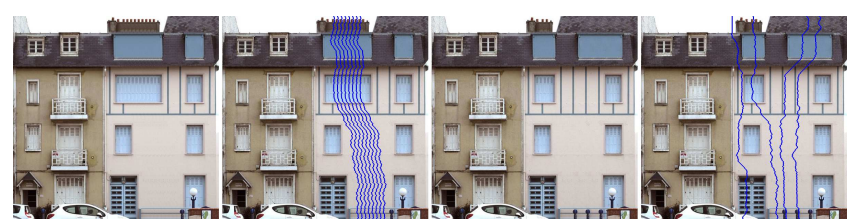

Figure 3: Enlarging from 512 to 600 pixels with $\xi=600$. Left: Without threshold $(R=\infty)$, the chimney appears ten times in a row. Right: With a threshold $R=4$, the result is visually improved.

edges exiting a node $(c, z)$ are deduced by storing with $c$ a pointer to its set of parallel cuts (see Eq.2).

\subsection{Limiting repetitions}

Our graph formulation above works well as such for synthesizing images of width smaller than the source. However, for larger images undesirable repetitions appear: in the result image, two nodes with the same cut appear repetitively at successive nearby locations (see Figure 3 ).

We implement a histogram-based scheme to avoid such behavior. It takes two parameters: a window size $\xi \in \mathbb{N}$ and a threshold $R \in$ $\mathbb{N}^{\star}$. We search a path in $G$ constrained so that in any crop of fullheight and width $\xi$ of its corresponding output image, no column of the source image is repeated more than $R$ times. Since most cuts are not vertical, a column in the result is not necessarily a column from the source image. We thus consider, as an approximation, that following a growth-edge $\left((c, z),\left(c^{\prime}, z^{\prime}\right)\right)$ copies the source columns with index in $\left(c_{\min }, c_{\min }^{\prime}\right]$ into the result. In Dijkstra's algorithm we do not follow an edge if it would result in a path not enforcing the constraint. An example of limiting repetitions is given in Figure 3 This limitation scheme is enabled by default in our implementation and all the results in the paper use $\xi=100$ and $R=2$, except where otherwise stated.

\subsection{Finding parallel cuts}

The construction of the set of cuts $\mathcal{C}$ is a crucial step for our technique. First, in terms of quality since we want parallel cuts with minimal cost. Second, in terms of performance since a larger number of cuts makes the graph larger and longer to build, and slows down the shortest paths search.

A cut in $\mathcal{C}$ that has no corresponding parallel cut is useless for our purpose, since it does not introduce new possible assemblies. We thus search for pairs of parallel cuts $\left(c, c_{\|}\right)$to be inserted in $\mathcal{C}$. Two main criteria guide us in our search for pairs. First, parallel cuts with a large cost are wasteful since the start-edges joining them will most often not be followed, and if followed would result in a noticeable mismatch. Second, we want to avoid an accumulation of low-cost parallel cuts in a same area of the image as this results in a large number of similar paths having similar costs. This increases the size of the graph significantly with no benefit. On the contrary, pairs of a given width should cover as much of the source image as possible. This variety helps the method finding paths even in constrained cases, for instance when user control is used (Section 5 .

For finding parallel cuts, we use the procedure given in pseudocode hereafter. In line 1 , we use $E_{I}^{\sigma}(x, y)=\|I(x, y)-I(x+\sigma, y)\|^{p}$. In line 2, $\pi$ is the minimal-cost $Y$-monotone path from the top to the bottom row of $E_{I}^{\sigma}$; it is computed using dynamic programming just as in [Efros and Freeman 2001]. In line 3, the two parallel cuts $\left(c, c_{\|}\right)$satisfy $\omega\left(c, c_{\|}\right)=\sigma$ and their cost $\delta\left(c, c_{\|}\right)$is that of the path $\pi$. Line 4 is the heuristic that we use to enforce that all the pairs of width $\sigma$ be well spread over the image while not being too

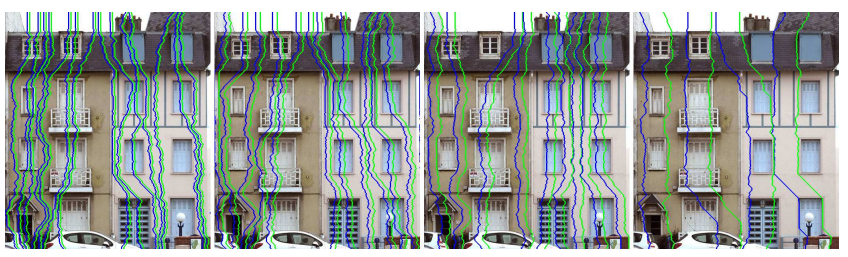

Figure 4: From left to right: Parallel cuts obtained for width $\sigma=$ 5,11,19 and 58. Each blue cut has a parallel green counterpart.

numerous (Figure 4). On a source image of size $256^{2}$, for example, the procedure typically finds around 600 pairs, with at least the best pair for each width.

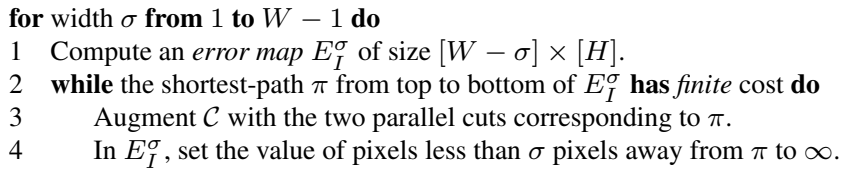

The preprocessing stage for one source image consists in computing a set of vertical cuts for horizontal resizing and a set of horizontal cuts for vertical resizing. These sets are stored on disk for later use.

\section{Bidirectional synthesis}

We described previously how to synthesize new images of arbitrary width or height. In most scenarios, however, a bidirectional synthesis is required. We rely on the fact that our synthesizer directly generates images of the desired width in one step: We apply two successive synthesis operations, one in each direction. The second synthesis step uses the intermediate result as a source image. This fits well with our goal of compactly encoding results in GPU memory, as only one vertical and one horizontal assembly are stored for each result. While the two possible orders of synthesis steps may produce different results, we observe that they are often very similar. All the results in this paper are obtained using vertical followed by horizontal synthesis.

After the first, vertical synthesis step, the vertical cuts for the horizontal synthesis have to be computed on the intermediate result. We provide a fast way to do so, as explained in Section 4.1 The graph for horizontal synthesis is then computed, and the second synthesis step proceeds. Section 4.2 explains how to accelerate the synthesis of many images of different sizes from a single source image.

\subsection{Fast cut update}

During bidirectional synthesis the computation of new cuts on the intermediate result becomes a bottleneck when synthesizing a large number of images. We propose an approximate but very fast way to obtain the cuts. We assume in the following vertical followed by horizontal synthesis. We discuss computation of vertical cuts for the second, horizontal synthesis step.

We observe that the vertical cuts computed as in Section 3.4 in the intermediate result tend to be locally identical to the vertical cuts in the source image. For instance, compare Figure 5 (b) and (d). We exploit this property to quickly compute new cuts. During the initial cut computation in the source image, we record for each width $\sigma$ a path map $P_{I}^{\sigma}$ in memory. It has the same size as the error map $E_{I}^{\sigma}$. Each entry $P_{I}^{\sigma}(x, y)$ is in $\{-1,0,1\}$ and indicates to which pixel to continue to reach the top of the error map with minimal cost. $P_{I}^{\sigma}$ is initialized using dynamic programming on the error map prior to the first path extraction (Section 3.4). After all vertical paths have been extracted we update $P_{I}^{\sigma}$ as follows: each pixel in $P_{I}^{\sigma}$ along a 


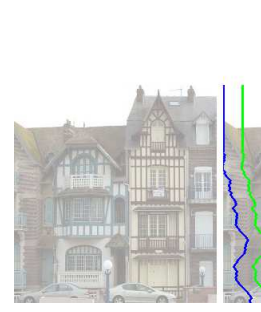

(a)

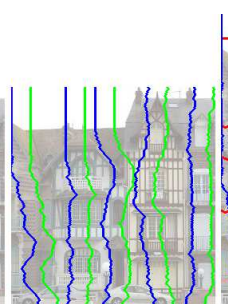

(b)

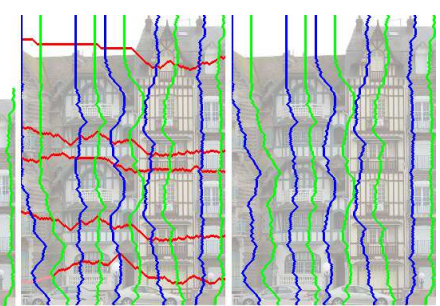

(c) (d)
Figure 5: Only parallel cuts of width $\sigma=24$ are shown for clarity. From left to right: (a) Source image, (b) precomputed parallel cuts for horizontal synthesis, (c) fast update of the vertical cuts; the cuts used for vertical synthesis are shown in red, (d) parallels obtained with exact computation on the intermediate result. The cuts in (c) and $(d)$ are very similar.

path is updated with the direction of that path at that position. We finally record the starting abscissa of each path. Thus, following $P_{I}^{\sigma}$ from these abscissa will reconstruct the same paths. Starting from any other location in $P_{I}^{\sigma}$ rapidly leads back to one of the paths.

The first, vertical synthesis step produces an assembly $A_{v}$ of horizontal strips from the source image. We apply $A_{v}$ to the path mapcutting it instead of the source image-to obtain a new path map $P^{\prime}$ corresponding to the intermediate image. Since the path map is narrower by $\sigma$ pixels than the source, we crop the cuts of $A_{v}$ to width $W-\sigma$. We then follow the directions given by $P^{\prime}$, starting from the recorded abscissa, to construct new paths from which we deduce pairs of parallel cuts just as in Section 3.4 (Figure 5(c)). We finally compute the cost of each pair using the intermediate result.

This process produces a new set of parallels in tens of milliseconds for all widths $\sigma$. It is approximate however, so there is no guarantee that the new paths are of minimal-cost in the error map of the intermediate result. However, since the cost of each new pair of parallel cuts is recomputed, bad pairs will tend to be avoided when traversing the graph $G$. Figure 5 compares the fast cut update to the computation described in Section 3.4

\subsection{Synthesizing in bulk}

Our goal is to generate, from a source image, a unique texture for many different surfaces. A good example of this scenario is the virtual city described in Section 7. where a small set of facade textures are customized to fit every facade of every building.

In this situation, we benefit from the fact that Dijkstra's algorithm computes a one-to-all shortest-paths tree: The vertical synthesis steps of all images start from the same node in $G$ (typically the top row of the source image). There are of course multiple end nodes, corresponding to the various requested heights. By keeping in memory the shortest-paths tree between successive calls to the Dijkstra's algorithm, we benefit from the previously computed shortest-paths. However, this cannot be done for the second synthesis step unless intermediate results are the same (i.e. same height, different widths). Nevertheless a significant speedup is achieved. For example, generating 10 images of height 588 and width ranging from 200 to 2000 takes 24.2 seconds in total if Dijkstra's algorithm is restarted from scratch for each image. Using our scheme, it takes 5.9 seconds to generate all the images.

\section{User control}

Our algorithm may operate automatically, but also provides several user controls over the synthesized images. In particular, the user may require some features to appear no more than a given number of times (Section 3.3), to be precisely localized (Section 5.1, , or may want a variety of results of a same size (Section 5.2). We focus below on the later two forms of control.

\subsection{Explicit feature positioning}

Explicit positioning lets the user specify where features of the source image should appear in the result. This is a convenient control for architectural textures. We discuss below two possible approaches: The first, simple and fast, lets the user directly manipulate cuts in the result. However, choosing the right cuts to position features may be difficult. The second, more elaborate, provides an accurate drag-and-drop control.

Splitting the path Using this simple control, the user explicitly specifies a set of control nodes in the graph $G$ that should be part of the output path. The control nodes are sorted and included in between the initial start and end nodes. Then, a shortest path is computed between each pair of consecutive nodes.

This approach has the advantage of simplicity, but the result strongly depends on which cuts are manipulated. Nevertheless, this control mechanism has proven useful in our experiments: visually good results are consistently achieved, and moving a control node around (i.e., changing $(c, z)$ to $\left.\left(c, z^{\prime}\right)\right)$ is fast since the shortestpaths trees generated with Dijkstra's algorithm are kept in memory (Section 4.2). Figure 7.bottom illustrates this type of control.

Drag-and-drop control Here, we seek a path of minimal cost in $G$, such that a region specified in the source appears at a desired location in the output. The user selects a source region $\mathcal{R}_{s}$ sandwiched between two special cuts $m$ and $M$ subject to

$m(y) \leq M(y)$ and $(m(y)=\infty \Leftrightarrow M(y)=\infty)$ for all $y \in[Y]$.

The user then specifies the abscissa $\Delta \in \mathbb{Z}$ where the region must be located in the output: Pixel colors at position $\{(x, y), m(y) \neq$ $\infty$ and $m(y)<x \leq M(y)\}$ in the source should appear at position $\left(x+\Delta-m_{\min }, y\right)$ in the synthesized image. Those target positions together form the target region $\mathcal{R}_{t}$.

We note $X$ the (possibly empty) set of nodes whose cut intersects $\mathcal{R}_{t}$ in the result, $L$ the set of nodes to the left of $\mathcal{R}_{t}(\prec m)$, and $R$ the nodes to the right $(\succ M)$. Assuming $L$ and $R$ are non empty, a satisfying path always starts with a node in $L$ and ends in $R$. In addition, it can only transition through $X(L \rightarrow X, X \rightarrow X$, $X \rightarrow R$ ) or from $L$ to $R$ via edges between well aligned nodes, that is nodes $(c, z)$ with $z=c_{\min }+\Delta$. This ensures that $\mathcal{R}_{t}$ contains the properly aligned selected part of the image. If $L$ (resp. $R$ ) is empty the starting (resp. ending) node belongs to $X$. In Dijkstra's algorithm loop, we discard edges that do not obey the above rules. The set of integers $z$ for which $(c, z) \in X$ is an interval since $\mathcal{R}_{t}$ is $Y$-monotone. We store these intervals to check in constant time if an edge can be followed or not. This easily extends to multiple control regions. Figure 1 (b) shows results obtained in this way.

\subsection{Introducing variety}

By default, our algorithm always generates the same image for given target size, histogram and user-control parameters. We implement an optional mechanism to automatically introduce variety as we successively synthesize new images from the same set of parameters. Let $C_{i}^{j}$ be the column of the source image that appears as the $j$-th column in the $i$-th result image (we make the same approximation as in Section 3.3. Successively synthesized images 
are forced to be different by ensuring that for all $j, C_{i}^{j}$ is farther than $D$ pixels away from $C_{i^{\prime}}^{j}$ for all $i^{\prime}<i$. That constraint is ignored for values of $j$ closer than $2 \times D$ pixels from the result image boundaries, since all results start and end similarly. We implement the constraint in Dijkstra's algorithm by ignoring edges which, if followed, would put two columns closer than allowed. Refer to Figure 8 (top) for results.

\section{Rendering}

We now describe how synthesized textures are applied to surfaces, having only the source image(s) and the assemblies describing the results in GPU memory. The result images are reconstructed onthe-fly, with proper filtering, in the pixel shader: we focus on rendering with a programmable GPU for real-time applications, but the technique applies equally well to off-line rendering.

Data structure In order to reconstruct a single result we only need to store the horizontal and vertical paths. Multiple results provide further opportunities for memory savings: Many of the precomputed cuts used in the first, vertical synthesis step are likely to appear in more than one result. However, the cuts generated for horizontal synthesis cannot generally be shared.

On the GPU we store all the cuts, vertical and horizontal, in a single 2D texture: the cut table. Since they correspond to different results, they have different lengths. We pack them together while heuristically minimizing wasted texture space. Each result is then encoded in two arrays, one for each path. For each node $(c, z)$ in a path we store in the array the index of the cut in the cut table, and its location $z$ in the result. Each pair of consecutive entries in the array defines a strip of the assembly.

Sampling and filtering During rendering, a small program - the pixel shader-is executed by the GPU at every pixel to determine its color. Each pixel is associated with a texture coordinate $u, v$. With standard texturing the texture image stored in memory is sampled at the $u, v$ location to obtain the pixel color.

Our goal is to find the color of each pixel using only the cuts, without ever having to decode the entire result image in memory. In each pixel we thus must find the coordinates $u_{\text {src }}, v_{\text {src }}$ in the source image corresponding to the texture coordinate $u, v$ in the result.

When performing a bidirectional synthesis, it is easy to see that the horizontal (resp. vertical) step changes only the $x$ (resp. $y$ ) coordinate of the pixel. Computations on $u$ and $v$ must be performed in the reverse order of synthesis to take into account the intermediate result: We obtain $u_{\text {src }}$ from $u, v$ and then $v_{\text {src }}$ from $u_{\text {src }}, v$. In a given direction, we first locate the strip enclosing the lookup coordinate in the result. We perform a binary search using the positions of the cuts. From there, we easily locate the source coordinates using the left strip border (see illustration hereafter).
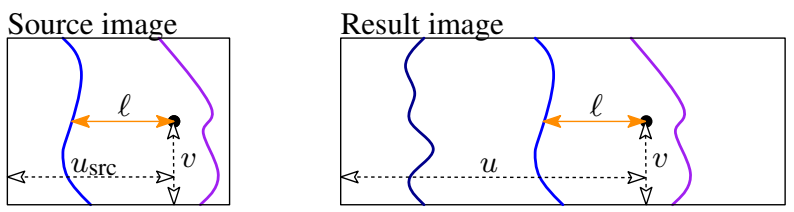

A key issue when rendering from a pixel shader is to ensure the result is filtered: the texture must be smoothly interpolated at closeups and properly blurred when seen at a distance (typically using MIP-mapping). The GPU filtering mechanism breaks around cuts since it is unaware of the discontinuities: visible seams appear. One approach would be to re-implement the trilinear interpolation mechanism. However, while feasible, this would be slow and would not support anisotropic filtering which is an important feature when rendering flat surfaces such as walls and facades. Instead, we blend the filtered lookups from both sides of a cut, hiding the interpolation seams. The blending weights are computed as a function of the distance to the cut and the filtering level. We treat both directions separately to enable support for anisotropic filtering. This removes all visible artifacts, even though discontinuities still exist at very coarse magnification levels where blending should involve multiple strips (please refer to the supplemental material).

\section{Applications and results}

We now present results and timings. All results are obtained on an Intel Xeon E5520 $2.3 \mathrm{GHz}$ using a single core for computations. The GPU is a NVidia Quadro FX 3800.

Synthesis of architectural textures We tested our approach on a number of architectural textures: floors, doors and window frames, fences and even structured photographs. The only parameter we change for these results is the number of allowed repetitions. We typically require no more than two repetitions in any crop a hundred pixels wide ( $\xi=100$ and $R=2$ ).

Figure 6 and 7 show a number of results, from simple ones to challenging cases. Table 1 gives timings for some of these images, including preprocessing for searching cuts-performed only once for a source image - and timings for synthesizing a new image. For each result image, we distinguish timings of each synthesis step and indicate how long the cut and graph update took. Note these are timings for the first result, when no shortest path has been previously computed. Subsequent results are faster to synthesize.

Result quality is overall very good, with very few visible discontinuities. Note that some remaining visible cuts in our results could be easily avoided by working in the gradient domain. However, this would impede compact encoding on the GPU.

Controlled Synthesis The user can directly specify where parts of the image should appear. This is illustrated Figures 1 (b) and 7 as well as in the accompanying video. Explicit positioning adds very little cost: precomputing the intervals for the drag-and-drop control takes 5 to 6 milliseconds, and the predicate that decides if an edge can be followed or not adds a negligible cost to the synthesis time.

City modeling The city modeling application reveals the key advantage of our approach compared to standard image resizing techniques. Since each synthesis result is compactly encoded, we are able to customize a facade texture to each and every surface of an entire city. Figure 1 shows a city with 7488 facades (1672 buildings), using 13 different texture images as sources (9.9 MB). Storing a texture for each facade would require $6.9 \mathrm{~GB}$ of GPU memory. Instead, using our scheme, each facade gets a unique texture and all facades occupy $60 \mathrm{MB}$ of GPU memory (excluding the source images); two orders of magnitude less. The textures are decoded in every pixel, from the pixel shader. The entire city renders at 195 FPS on average at $1600 \times 1200$ resolution, without deferred shading or visibility culling. All computations for the facades took

\begin{tabular}{|c|c|c|c|c|c|c|}
\hline Source & Preproc. & \multicolumn{5}{|c|}{ Synth. = V synth. + Cuts + Graph + H synth. } \\
\hline Fig. 6 right $(256 \times 256)$ & 2.5 & 0.8 & 0.06 & 0.02 & 0.19 & 0.5 \\
\hline Fig. 7 A $(340 \times 512)$ & 20.3 & 5 & 3 & 0.1 & 0.9 & 1 \\
\hline Fig. 7 B $(512 \times 512)$ & 22.7 & 6.7 & 1 & 0.16 & 2.3 & 3.2 \\
\hline Fig. 7 C $(512 \times 349)$ & 21.8 & 5.4 & 0.88 & 0.1 & 2 & 2.4 \\
\hline
\end{tabular}

Table 1: Timings in seconds for various syntheses. 

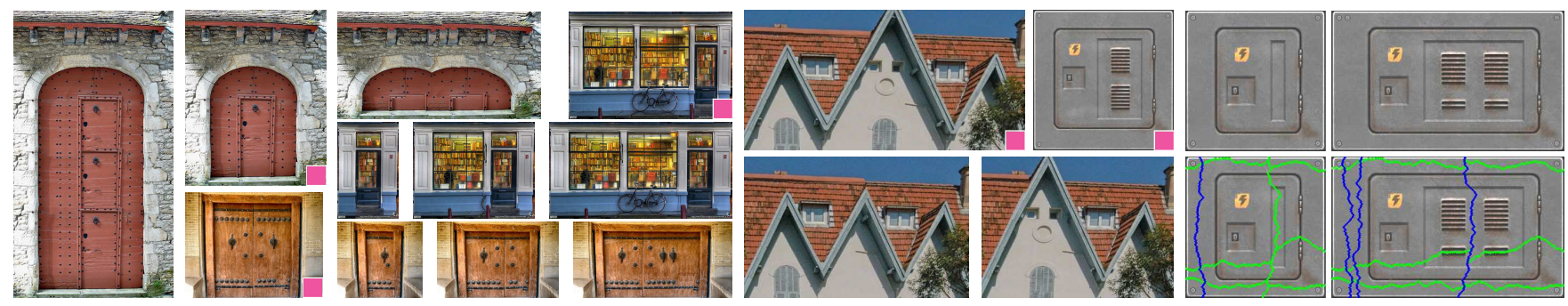

Figure 6: Various syntheses. Sources are marked with a pink square. Right: Source is slightly downscaled, bottom results have cuts overlaid.

78 minutes, for an average of $625 \mathrm{~ms}$ and $8 \mathrm{~KB}$ per facade. As can be seen on these images as well as on the accompanying video, filtering is well behaved, showing no artifact even at grazing angles. Anisotropic filtering is enabled in all renderings.

Comparison to grammar-based facade synthesis The approach of [Müller et al. 2007] fits a grammar on a facade image, enabling procedural generation of new facades. It operates at the semantic level, explicitly detecting rectangular shaped windows and doors. In contrast, our approach operates at a lower level and does not require a description of the image content. A drawback is that it may produce semantically improper results such as narrow or asymmetric doors or windows. However, it applies in a larger number of cases such as decorated, complex, partially occluded or unusual facades (see Figures 6 and 7). Our approach complements well grammar-based techniques, providing a good alternative when the grammar cannot interpret the image content.

Variety Figure 8 (top) shows an example of introducing variety using our algorithm. Four successive results of size equal to the source are synthesized. The leftmost image - the first result-is exactly similar to the source. All three successive images are constrained to be different from the previous ones.

Comparison to retargeting Our approach synthesizes a variety of images of different sizes resembling the input, while retargeting generates images preserving all the content visible in the original. In Figure 8 (bottom) we compare our approach with that of Avidan and Shamir [2007] and Simakov et al. [2008] for enlargement. On the more elaborate scheme of Simakov et al. results are very similar. However we have the possibility to quickly generate a variety of new, different images. In addition, the result of retargeting approaches are new images which cannot be compactly stored during rendering. Of course, latest retargeting approaches do not require our strong assumption about the content, and there are many images we could not deal with.

Limitations The main limitation of our approach is that it focuses on architectural textures, expecting repetitions to occur mostly along the $\mathrm{x}$ and $\mathrm{y}$ directions. Figure 9 shows two failure cases. In both, either no repetition exist or the repetitions are not aligned with the main axes producing artifacts in the results. As mentioned earlier, our method has no semantic information and may produce unrealistic features (such as narrow doors, long cars).

Another limitation is that a path may not always exist when performing user control, or the path found may have a very large error. Using arbitrarily shaped curves rather than $Y$-monotone curves would add more freedom and allow for more complex behaviors. The graph formulation does not have to be changed, but this complicates storing and decoding the cuts in the GPU. We leave this issue for future work.

Finally, on extreme enlargements large scale repetitions may ap-
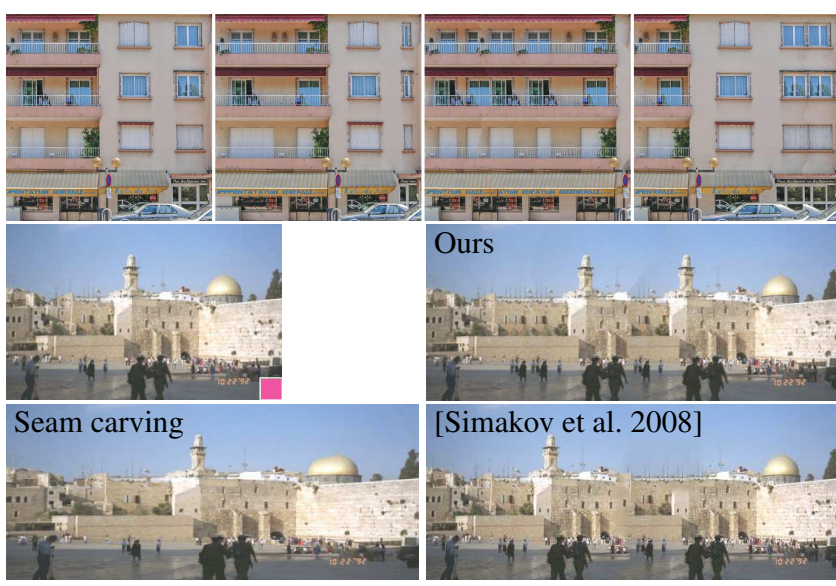

Figure 8: Top row: Four results of same size with variety. ( $D=$ $48, R=3, \xi=\infty)$. Bottom: Comparison with retargeting.
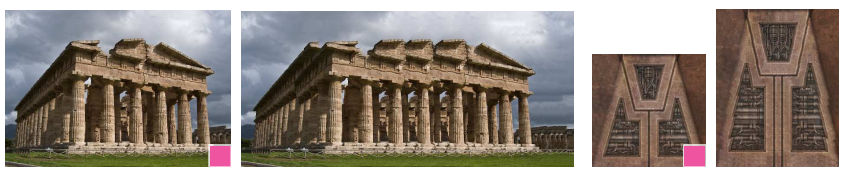

Figure 9: Left pair: Source image [Barnes et al. 2009], our result. Right pair: source without vertical repetition, our result.

pear, producing a distracting tiling effect. As future work, we plan to implement a mechanism similar to the one for variety but within a same image, so as to avoid these large scale repetitions.

\section{Conclusion}

Our approach significantly reduces authoring time of large environments while preserving rendering performance, both in time and memory consumption. Each support surface is fitted with a texture of the correct size and aspect-ratio, thereby improving the overall visual quality. The technique appears robust and fast enough to be used as an interactive and creative tool for the transformation of texture assets in virtual worlds.

We believe the graph based formulation of our approach is especially well-suited for synthesizing structured content decomposed in basic blocks, while enforcing non trivial user constraints. In particular, it is easy to generalize the formulation to any other type of content as long as synthesis can be decomposed in a set of successive unidirectional operations (not necessarily axis aligned or along a straight line). We hope this will spark further research in the field of by-example content synthesis.

Acknowledgments and Credits We thank Laurent Alonso, George Drettakis, Bruno Lévy, Nicolas Ray and Rhaleb Zayer for proof-reading the paper. Credits for the source images go to: Claire Royer (Figure 7 top right), Asmodai Tabun (video game textures, Figures 6 7 9, Denyse Schwab (Mandala, Figure 7), Yaron Caspi (Jerusalem, Figure 8), Flickr users Sev- 

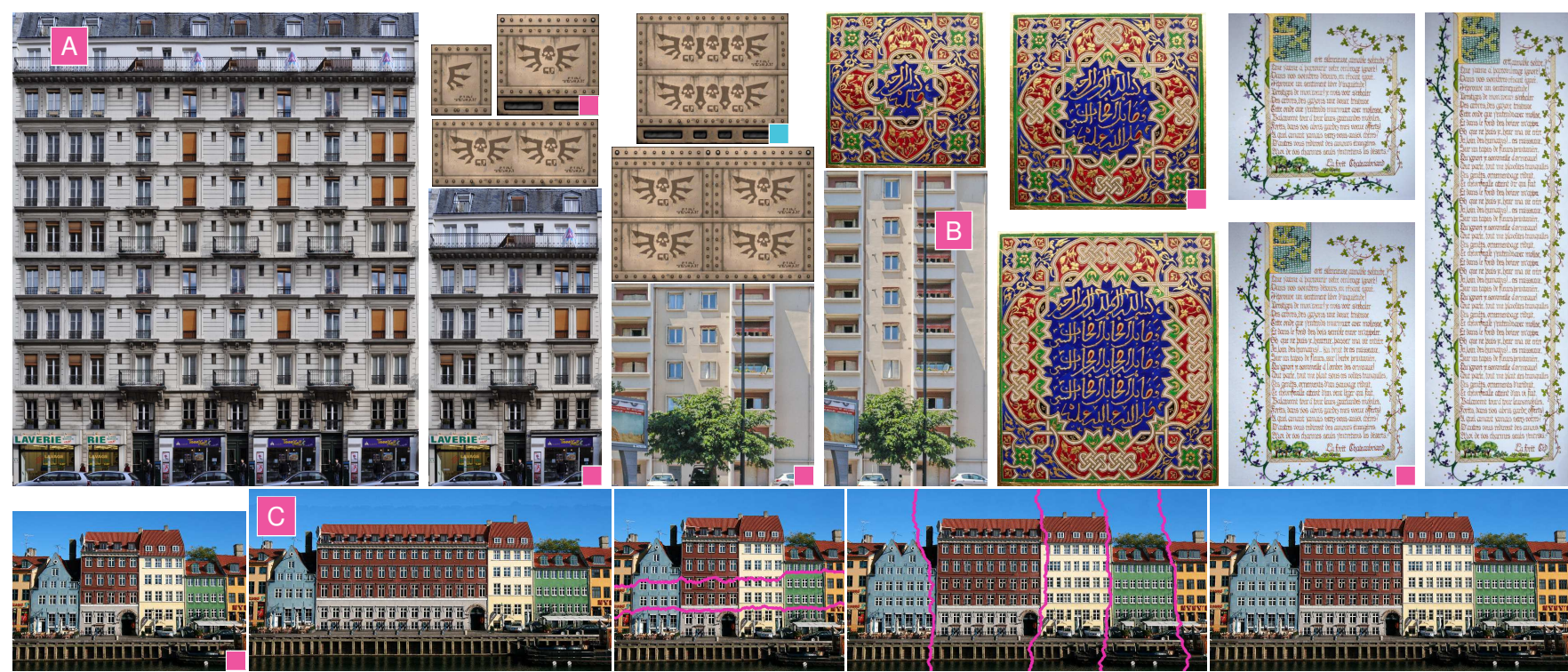

Figure 7: Top: Synthesis results. Source images are marked with a pink square. The histogram window size parameter $\xi$ is 250 for the leftmost image. Drag-and-drop control is used on the image marked with a blue square. Bottom, left to right: The Copenhagen harbor. Automatic enlargement. 2 control cuts (in pink) to add one floor. 4 control cuts to set the building sizes. Final result.

enbrane (greek temple, Figure 9, Dynamosquito (doors, Figure 6, Bcnbits (bookstore, Figure 6), www. cgtextures.com (Figure] (a (middle), b), 3 4 5, [Teboul et al. 2010] (Figure 1] (a (left, right)), Figure 7]A), Centre Scientifique et Technique du Bâtiment (all other facades). This work was funded by the Agence Nationale de la Recherche (SIMILAR-CITIES ANR-2008-COORD-021-01) and the European Research Council (GOODSHAPE FP7-ERC-StG-205693).

\section{References}

Avidan, S., AND Shamir, A. 2007. Seam carving for contentaware image resizing. ACM Transactions on Graphics (Proc. ACM SIGGRAPH) 26, 3.

Barnes, C., Shechtman, E., Finkelstein, A., And GoldMAN, D. B. 2009. PatchMatch: A randomized correspondence algorithm for structural image editing. ACM Transactions on Graphics (Proc. ACM SIGGRAPH) 28, 3.

Cabral, M., Lefebvre, S., Dachsbacher, C., ANd DretTAKIS, G. 2009. Structure preserving reshape for textured architectural scenes. Computer Graphics Forum (Proc. Eurographics conference) $28,2$.

Cho, T. S., Butman, M., Avidan, S., and Freeman, W. T. 2008. The patch transform and its applications to image editing. In Proc. IEEE Conference on Computer Vision and Pattern Recognition (CVPR).

Efros, A. A., And Freeman, W. T. 2001. Image quilting for texture synthesis and transfer. In Proc. ACM SIGGRAPH, 341346.

GAL, R., Sorkine, O., AND COHEN-Or, D. 2006. Featureaware texturing. In Rendering Techniques, Proc. Eurographics Symposium on Rendering, 297-303.

Kwatra, V., Schödl, A., Essa, I., Turk, G., AND Bobick, A. 2003. Graphcut textures: Image and video synthesis using graph cuts. ACM Transactions on Graphics (Proc. ACM SIGGRAPH) 22, 3.

Lefebvre, L., AND Poulin, P. 2000. Analysis and synthesis of structural textures. In Proc. Graphics Interface (GI), 77-86.

Legakis, J., Dorsey, J., AND Gortler, S. 2001. Featurebased cellular texturing for architectural models. In Proc. ACM SIGGRAPH, 309-316.
Müller, P., Zeng, G., Wonka, P., And Gool, L. V. 2007. Image-based procedural modeling of facades. ACM Transactions on Graphics (Proc. ACM SIGGRAPH) 26, 3.

Pritch, Y., KaV-Venaki, E., And Peleg, S. 2009. Shiftmap image editing. In Proc. IEEE International Conference on Computer Vision (ICCV).

Rubinstein, M., Shamir, A., And Avidan, S. 2009. Multioperator media retargeting. ACM Transactions on Graphics (Proc. ACM SIGGRAPH) 28, 3.

SchöDl, A., Szeliski, R., SAlesin, D., AND EsSA, I. 2000. Video textures. In Proc. ACM SIGGRAPH, 489-498.

Simakov, D., Caspi, Y., Shechtman, E., And Irani, M. 2008. Summarizing visual data using bidirectional similarity. In Proc. IEEE Conference on Computer Vision and Pattern Recognition $(C V P R)$.

Sun, J., YUAN, L., JiA, J., AND SHUM, H.-Y. 2005. Image completion with structure propagation. In Proc. ACM SIGGRAPH, 861-868.

Teboul, O., Simon, L., Koutsourakis, P., And Paragios, N. 2010. Segmentation of building facades using procedural shape priors. In Proc. IEEE Conference on Computer Vision and Pattern Recognition (CVPR).

WAnG, Y.-S., TAI, C.-L., Sorkine, O., AND LEE, T.-Y. 2008. Optimized scale-and-stretch for image resizing. ACM Transactions on Graphics (Proc. ACM SIGGRAPH Asia) 27, 5.

Wei, L.-Y., Lefebvre, S., KWATrA, V., AND Turk, G. 2009. State of the art in example-based texture synthesis. In Eurographics 2009, State of the Art Report, EG-STAR, Eurographics Association.

Worley, S. P. 1996. A cellular texturing basis function. In Proc. ACM SIGGRAPH, 291-294. 